\title{
Tratamiento quirúrgico del carcinoma adenoideo quístico de la tráquea. Presentación de un caso y revisión de la literatura
}

\author{
Marco Antonio Iñiguez-García, $\bowtie$ Enrique Guzmán-de-Alba, César Luna-Rivero, \\ Gustavo Félix Salazar-Otaola, Carlos Manuel Núñez-Bustos, Juan Carlos Vázquez-Minero
}

Instituto Nacional de Enfermedades Respiratorias Ismael Cosío Villegas, Ciudad de México.

Trabajo recibido: 14-IV-2014; aceptado: 07-V-2014

\begin{abstract}
RESUMEN. Introducción: Los tumores malignos primarios de tráquea son raros, en el adulto representan el $90 \%$ de todos los tumores traqueales. El carcinoma adenoideo quístico es el segundo más frecuente con aproximadamente del 10-15\% de los casos. Los síntomas son inespecíficos y los más frecuentes suelen ser: tos, ronquera, disnea, sibilancias y estridor. La broncoscopia es el método para la obtención de tejido para el estudio histológico. La resección quirúrgica es el tratamiento de elección siempre que sea posible. Métodos: Se presenta el caso de una mujer de 59 años de edad con diagnóstico de carcinoma adenoideo quístico en tercio medio de la tráquea, a quien se le realizó resección traqueal con anastomosis terminoterminal, obteniendo resección completa, no recibió adyuvancia, con seguimiento de 15 meses sin recurrencia. Discusión y conclusiones: El manejo de pacientes con carcinoma adenoideo quístico debe ser multidisciplinario. A la paciente se le pronosticó una tasa de sobrevida a 5 años de $91 \%$ por tratarse de una enfermedad localizada. El diagnóstico de este tipo de tumores tiene un subregistro y el manejo quirúrgico está subutilizado en México. Los pacientes con este tipo de tumores, en particular, y todos los tumores traqueales, en general, deben ser referidos a centros con experiencia para el manejo de patología traqueal.
\end{abstract}

Palabras clave: Carcinoma adenoideo quístico, resección de tráquea.

ABSTRACT. Introduction: Primary malignant tumors of the trachea are rare, but they represent $90 \%$ of all tumors of the trachea. The adenoid cystic carcinoma is the second most frequent hystologic type of tumor growing in the trachea with aproximately 10 to $15 \%$ of all cases. Symptoms are unspecific and the most frequent are cough, hoarseness, dyspnea, wheezzing and stridor. Bronchoscopy is the study of choice to obtain tissue for histopathologic study. Surgery is the treatment of choice when possible. Methods: We present the case of a 59 years old female with an adenoid cystic carcinoma of the middle third of the trachea, treated with surgical resection, obtaining a complete resection, with no adyuvant therapy, and with 14 months follow-up without recurrence. Discussion and conclusions: Treatment of adenoid cystic carcinoma should be multidisciplinary. In our patient had forecast a rate of 5-year survival of $91 \%$ because it was a localized disease. We consider that patients with any type of tracheal tumor should be referred to a specialized center with experience in the treatment of tracheal pathology.

Key words: Adenoid cystic carcinoma, tracheal resection.

\section{INTRODUCCIÓN}

Los tumores malignos primarios de tráquea son raros, la incidencia reportada en Finlandia, Dinamarca y los Países Bajos es de 0.1 por 100,000 habitantes por año; la frecuencia del cáncer traqueal es menor a $0.5 \%$ de todos los tumores malignos. Aproximadamente se presentan en una proporción de 1:180 con respecto al carcinoma broncogénico sin conocer, hasta la fecha, cuáles son los mecanismos de defensa de la mucosa traqueal frente a los agentes carcinógenos. En el 70\% de los casos son hombres y el promedio de edad de presentación es de 60 años. ${ }^{1-3}$
La serie más grande reportada es de 578 casos en un período de 31 años por The Surveillance Epidemiology and End Results (SEER), programa del Instituto Nacional del Cancer $(\mathrm{NCl}$, por sus siglas en inglés) de Estados Unidos de América. En esta serie, el $56 \%$ eran hombres y la media de edad de presentación de 63 años. ${ }^{4}$

En el adulto los tumores traqueales malignos representan el $90 \%$ de todos los tumores traqueales. El carcinoma de células escamosas es el tumor traqueal más frecuente (45-65\%); y el segundo más común, es el carcinoma adenoideo quístico (CAQ) con aproximadamente del $10-15 \% .^{1-4}$ De acuerdo al origen, los tumores 
traqueales se clasifican en epiteliales, mesenquimatosos y linfomas. A su vez, los epiteliales están clasificados en carcinomas, papilomas, neuroendocrinos y de glándulas salivales. A este último grupo pertenece el adenoideo quístico. ${ }^{5}$

EI CAQ de la tráquea es idéntico al carcinoma adenoideo quístico de las glándulas salivales. Este tipo de tumores típicamente forma lesiones polipoides en la tráquea o en los bronquios principales; pero pueden formar placas infiltrativas con extensión longitudinal o circunferencial, y a menudo incluso romper la capa cartilaginosa.

En 1859, Billroth describió el CAQ, anteriormente denominado cilindroma porque su aspecto macroscópico recordaba a un tumor benigno que respeta la integridad de la mucosa. ${ }^{6}$ La invasión perineural y extensión a lo largo de estructuras vasculares es muy común y presenta una tasa alta de márgenes quirúrgicos positivos, ${ }^{7,8}$ puede tener múltiples recurrencias con metástasis tardías.

Para su diagnóstico la clínica no es muy útil, ya que los síntomas son inespecíficos, siendo los más frecuentes: tos, ronquera, disnea, sibilancias y estridor. Muchos pacientes son inicialmente mal diagnosticados como $p$. ej., asma o infección de vías respiratorias, o bien cáncer de tiroides invasivo, entre otros. ${ }^{9}$

La radiografía de tórax rara vez es útil para el diagnóstico. La tomografía computada (TC) de tórax con reconstrucciones multiplanares es un excelente método de formación de imágenes y detección de las lesiones traqueales. La espirometría con curvas flujo-volumen puede demostrar el aplanamiento de la curva en la inspiración (tumor extratorácico) o en la espiración (tumor intratorácico) o ambos, si existe afectación sustancial de la tráquea; aunque este estudio es limitado en lesiones pequeñas. La broncoscopia es el método de elección para obtener tejido para el estudio histológico y es muy útil en la evaluación de la posibilidad de resección del tumor.

Estadificación. La tomografía con emisión de positrones con tomografía computada (PET-CT, por sus siglas en inglés), puede ser útil en la estadificación del carcinoma de células escamosas traqueal.

No hay un sistema de clasificación internacional. Un sistema de clasificación que ha sido utilizado por algunos investigadores se basa en el sistema TNM (tumor, nodo, metástasis), derivado de la base de datos de la SEER..$^{10}$ La principal limitación del TNM en este tipo de tumores, es el pequeño número de casos, lo que genera información insuficiente para permitir una evaluación precisa de su capacidad para predecir la supervivencia.

Las modalidades principales de tratamiento son la cirugía y la radioterapia (RT), aunque no hay ensayos aleatorios. La resección quirúrgica es el tratamiento de elección para estos tumores siempre que sea posible; sin embargo, la resección quirúrgica parece estar subutilizada de acuerdo a un estudio. ${ }^{11}$ Aunque hay series que presentan mayores índices de resecabilidad. En la serie del Massachusetts General Hospital 191 de 270 pacientes $(71 \%)$ con diagnóstico de carcinoma de células escamosas o carcinoma adenoideo quístico fueron resecables. ${ }^{12}$ La tasa de mortalidad operatoria a 30 días es reportada entre el 7 al 11\%,7,13,14 observándose una disminución en las últimas cuatro décadas de $3 \%$ en el período de 1992-2001.

La RT se aplica comúnmente a pacientes con resección incompleta o márgenes positivos (R1, R2). El beneficio del manejo con quimioterapia (QT) más RT es incierto, ya que no hay buenos estudios clínicos. ${ }^{15}$ No está claro si la QT modifica la historia natural de las metástasis a distancia.

La broncoscopia rígida con resección parcial y/o la utilización de cánulas traqueales pueden ser utilizadas mientras se realiza la cirugía. Estos recursos pueden emplearse como parte del tratamiento paliativo en tumores irresecables, aunque los beneficios son temporales. ${ }^{16-19}$

\section{MÉTODOS}

Se realizó la búsqueda en PubMed en noviembre de 2013 con los siguientes filtros (carcinoma, adenoid cystic [Mesh]) and trachea [Mesh] filters: Humans, english, spanish. Obteniendo un total de 59 artículos; de éstos, fueron descartados 38 artículos por tratarse de casos de tumores con involucro de estructuras adyacentes, lesiones en la carina, algunas reconstrucciones con material protésico, algunos manejados con resecciones pulmonares, resecciones traqueales por patología benigna. Finalmente quedaron 21 artículos, mismos que fueron los más significativos relacionados con la presentación de este caso.

\section{CASO CLÍNICO}

Mujer de 59 años de edad, originaria del Estado de México, con antecedentes de hipertensión arterial sistémica con 25 años de diagnóstico, apendicectomía y colecistectomía a los 31 y 54 años, respectivamente, exposición a biomasa por 12 años durante su infancia.

Su padecimiento actual lo inicio seis meses previos a su ingreso con disnea, estridor, con diagnóstico de asma por otra institución, tratada con broncodilatadores, esteroides, con mejoría parcial. La disnea progresó y el estridor en un inicio inspiratorio fue, posteriormente, bifásico con accesos de tos no productiva, con utilización 




Figura 1. Imagen endoscópica de la tráquea donde se observa (flechas) el tumor exofítico que ocasiona disminución de la luz traqueal.

de músculos accesorios para la ventilación. Ingresó al Servicio de Urgencias del Instituto Nacional de Enfermedades Respiratorias Ismael Cosío Villegas (INER), Ciudad de México, con datos de estenosis traqueal; motivo por el cual se le realizó broncoscopia flexible, observado tumor traqueal blanco nacarado, irregular (figura 1) desde el 2do. al 8vo. anillo traqueal (AT), 5 AT libres a la carina principal (CP) que ocasionaba disminución de la luz traqueal en un $90 \%$ y se dejó con intubación orotraqueal para permeabilizar la vía aérea. A los dos días le fue realizada una broncoscopia rígida con resección parcial de la tumoración para permeabilizar la vía aérea y toma de biopsia. En el posquirúrgico inmediato presentó enfisema subcutáneo en cuello, cara, tórax y neumotórax bilateral, colocándole sonda torácica bilateral. Por broncoscopia se identificó lesión traqueal, localizada en cara posterior en la unión con cara lateral de $1 \mathrm{~cm}$ de longitud a $3 \mathrm{~cm}$ de la $\mathrm{CP}$, se dio manejo conservador con intubación orotraqueal ferulizando la lesión traqueal, con ventilación mecánica invasiva por siete días. El Servicio de Patología del INER reportó carcinoma adenoideo quístico. Para estadificarla se realizó TC de tórax (figura $2 a, 2 b$ ), abdomen, pelvis y cráneo. Fue identificada la tumoración traqueal en tercio medio de la tráquea sin datos de lesiones a distancia. Se retiraron las sondas torácicas.

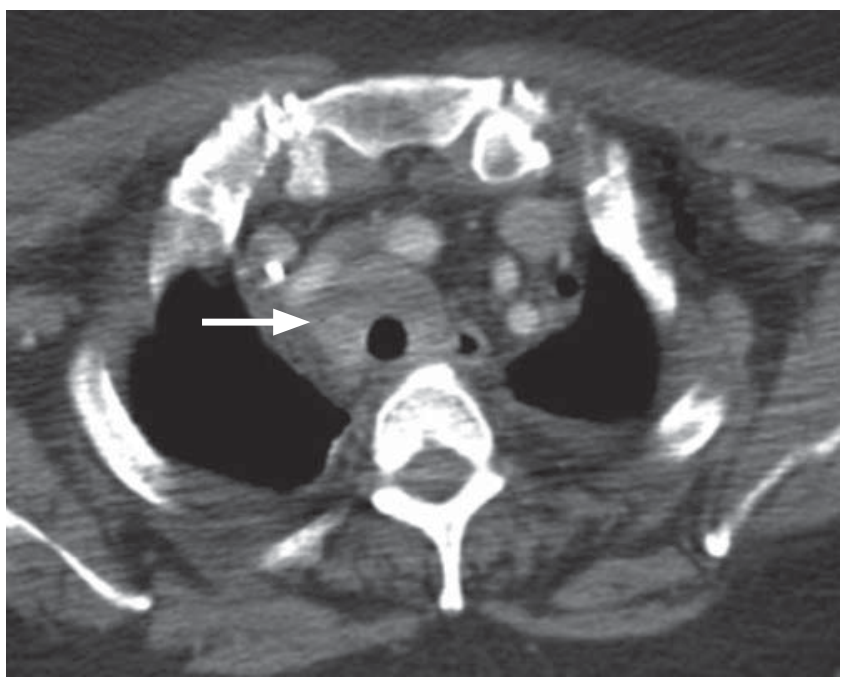

Figura 2a. TC de tórax contrastada de corte axial donde se observa (flecha) el tumor traqueal que disminuye la luz y está en contacto con estructuras vecinas sin invadirlas.

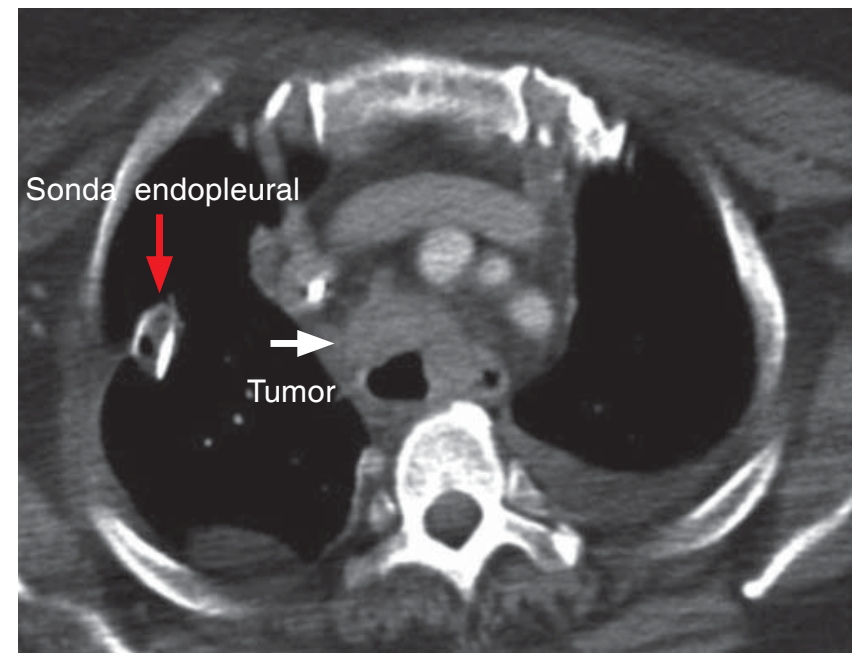

Figura 2b. TC de tórax contrastada de corte axial más inferior donde se aprecia tumor traqueal (flecha blanca), que disminuye la luz y está en contacto con estructuras vecinas sin invadirlas, además, de derrame pleural bilateral y la sonda endopleural derecha (flecha roja).

\section{Abordaje quirúrgico}

El abordaje cervical para la resección fue planeado, la paciente permaneció en decúbito dorsal para realizar la broncoscopia preoperatoria, se administró anestesia general balanceada con intubación orotraqueal, con cánula número 6 Fr., se colocó sonda esofágica $20 \mathrm{Fr}$. Se colocó con hiperextensión cervical. La disección fue 


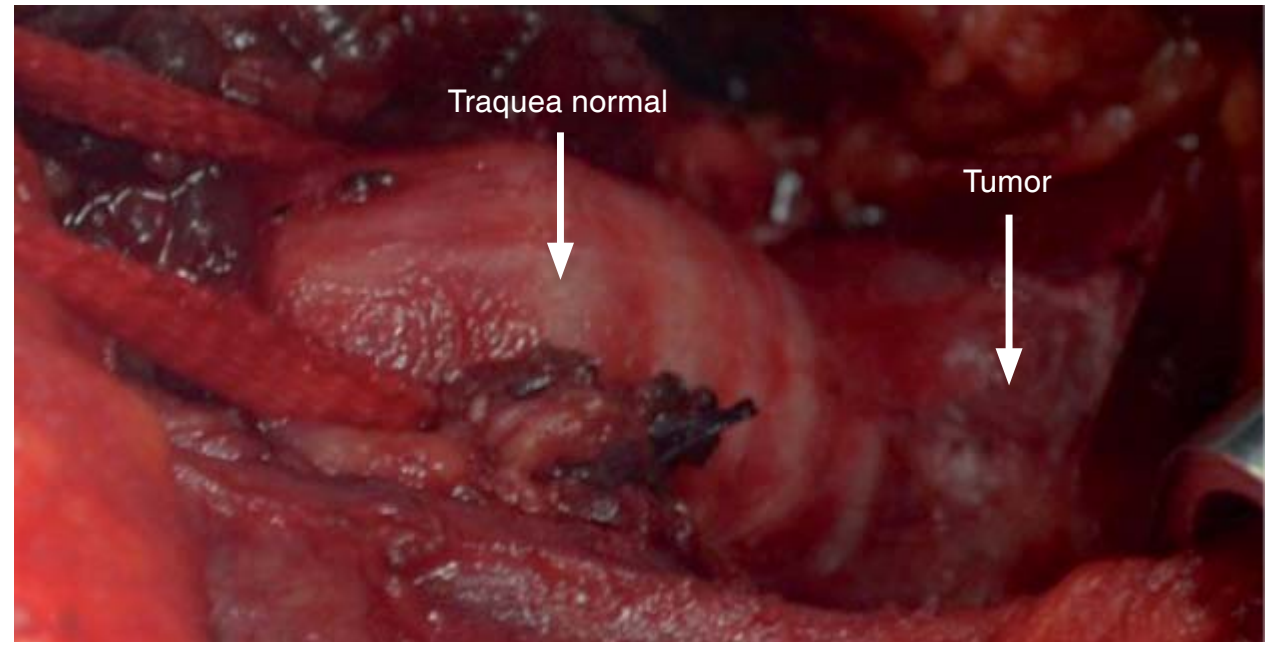

Figura 3a.

Se observa la tráquea expuesta haciendo tracción en sentido cefálico (lado derecho de la imagen) con una cinta de lino y el inicio del tumor hacia el lado izquierdo de la imagen.

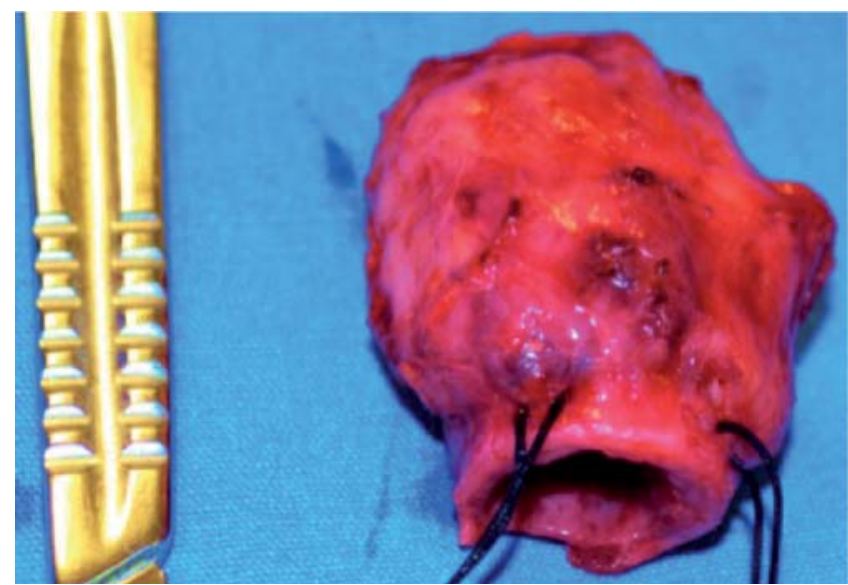

Figura 3b. Nótese la pieza quirúrgica completa con los puntos de sutura en la parte proximal.

a través de la línea media del cuello, el istmo se seccionó del tiroides para exponer la tráquea. La tráquea fue disecada hasta la carina principal para facilitar la movilización del segmento a resecar, se colocaron puntos de sutura distal y proximal al tumor para poder traccionar y manipular la tráquea, dejando el espacio suficiente para realizar la anastomosis. La tráquea fue cortada distalmente, la cánula orotraqueal retirada previa colocación de una sutura en el orificio de Murphy para facilitar la recolocación de la cánula después de haber realizado la anastomosis en la cara posterior; se cortó la tráquea en la parte proximal abarcando la totalidad del tumor (figura $3 \mathrm{a}, 3 \mathrm{~b}$ ), acto seguido fueron cortados bordes para enviarlos al estudio histopatológico transoperatorio. Para la ventilación cruzada por el campo quirúrgico, se utilizó una segunda cánula traqueal número 7.5 Fr., canulando la tráquea distal. El reporte

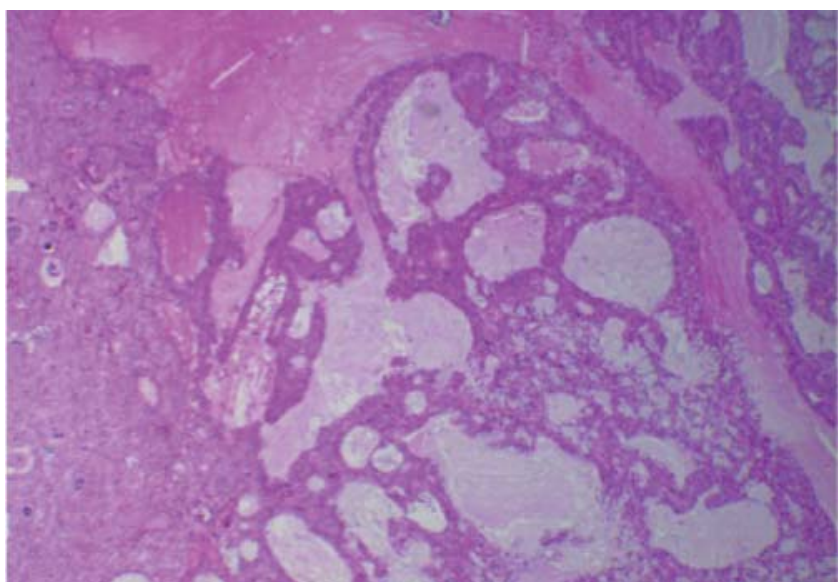

Figura 4. Neoplasia con patrón de crecimiento sólido-quístico, las áreas sólidas son densas, constituidas por abundantes células, las áreas quísticas muestran material mucinoso, en la periferia se observan cristales de colesterol (HyE 10x).

histológico fue: bordes libres de tumor. Para realizar la anastomosis traqueal se utilizaron puntos simples con sutura absorbible 3-0; después de anudar los puntos en la pars flácida se avanzó la cánula orotraqueal una vez retirada la segunda cánula. Finalmente fue concluida la anastomosis, verificada la ausencia de fuga aérea y realizada la hemostasia; se afrontaron los tejidos y se colocó un punto de sutura del mentón al tórax para la flexión cervical forzada durante seis días. Al segundo día posquirúrgico se movilizó fuera de cama. La paciente fue egresada al séptimo día posquirúgico y valorada por el Servicio de Oncología quien decidió no otorgar ninguna terapia adyuvante.

El reporte histopatológico de la resección quirúrgica mencionó carcinoma adenomatoideo quístico traqueal 
de $3.7 \mathrm{~cm}$ en su diámetro mayor con invasión perineural y linfovascular, bordes quirúrgicos sin tumor. Ganglios linfáticos con hiperplasia linfoide folicular y congestión sinusoidal (figura 4). Actualmente con 15 meses de seguimiento la paciente presenta buena evolución.

\section{DISCUSIÓN}

El caso presentado, mujer de 59 años de edad; en cuanto al género, la literatura reporta a los tumores malignos de tráquea más comúnmente en hombres hasta en un $70 \%$, pero un $30 \%$ se presentan en mujeres; y en cuanto a la edad promedio referido en las series ésta es de 60 años, lo que coincide con nuestro caso.

La paciente ingresó con datos de obstrucción de la vía aérea, razón para realizar broncoscopia y decidir dejarla con intubación orotraqueal y, así, permeabilizar la vía aérea; después, se le realizó broncoscopia rígida para resección parcial del tumor y llevar la muestra a patología y permeabilizar la vía aérea. El objetivo se logró, pero lamentablemente la tráquea fue perforada y como aún no había diagnóstico se decidió un manejo conservador con ventilación mecánica invasiva logrando ferulizar la lesión.

El uso de broncoscopia rígida con resección parcial y/o colocación de férulas traqueales de forma temporal, está reportado como puente para la cirugía. ${ }^{16-19}$ La literatura menciona que el factor más importante que influye en el pronóstico, es la extensión de la enfermedad al momento del diagnóstico. ${ }^{20}$ De acuerdo a la base de datos de la SEER, de 578 pacientes con tumores malignos de tráquea, en general, presentan una sobrevida a 5 años: con enfermedad localizada $47 \%$, enfermedad regional $26 \%$ y con metástasis a distancia $4 \% .{ }^{4}$ Se reporta que otro factor con mucho impacto en el pronóstico es la histología del tumor. Además, que la tasa de sobrevida a 5 años para el carcinoma adenoideo quístico es del $74 \%$, frente al $13 \%$ de carcinoma de células escamosas; y si solo tomamos en cuenta lo que presenta enfermedad localizada, ésta es del $91 \%$ frente al $25 \%$. Con base en lo anterior, a nuestra paciente se le pronostica una tasa de sobrevida a 5 años de $91 \%$, pues se trata de una enfermedad localizada con histología de CAQ.

En nuestra paciente se logró la resección Ro y acordamos no dar manejo adyuvante de acuerdo con lo reportado en la literatura. Es este aspecto, lo que encontramos reportado en varias series que incluyen un número significativo de pacientes con carcinoma adenoideo quístico. ${ }^{7,12,13,19,21}$ En una serie de Estados Unidos fueron reportados 101 pacientes con diagnóstico de carcinoma adenoideo quístico y resecados, el $70 \%$ de estos pacientes recibió RT postoperatoria. La tasa de sobrevida a los 5 y 10 años fue de 52 y $29 \%$, respectivamente. Y 34 pacientes fueron irresecables con una tasa se sobrevida a 5 años de $33 \% .^{12}$ Una serie francesa reportó 60 pacientes con CAQ con resección quirúrgica, de los cuales 26 (43\%) recibieron RT adyuvante con una tasa de sobrevida a los 5 y 10 años de, 73 y $57 \%$, respectivamente..$^{13}$ Una serie canadiense reportó 38 pacientes con CAQ, de los cuales 32 se resecaron y de éstos 26 fueron tratados con RT adyuvante. La tasa de sobrevida a los 5 años de los pacientes resecados, fue de $79 \%$ y a los 10 años de $51 \% .^{7}$

En un estudio que compara los resultados de 270 pacientes con carcinoma de células escamosas y carcinoma adenoideo quístico, el análisis multivariable observó que el CAQ se asoció a un mejor pronóstico. Entre los factores asociados con mejor resultado destacan la resección completa y los márgenes microscópicos negativos (R0)..$^{14}$

\section{CONCLUSIONES}

El manejo de pacientes con carcinoma adenoideo quístico debe ser multidisciplinario. Para los pacientes con CAQ de la tráquea se recomienda la resección quirúrgica completa cuando ésta sea posible (Grado 2B). La RT se da con frecuencia a los pacientes con resección incompleta o márgenes positivos. (R1, R2). No está claro el uso de RT para los pacientes $\mathrm{R} 0$ ya que no hay ensayos aleatorios. Cuando se trate de tumores irresecables se sugiere la $\mathrm{RT}$ o la $\mathrm{QT}$ combinada con un régimen basado en platino (Grado $2 \mathrm{C}$ ).

Consideramos que el diagnóstico de estos tumores tiene un subregistro, y el manejo quirúrgico en México está subutilizado. Además, que los pacientes con este tipo de tumores, en particular, y todos los tumores traqueales, en general, deben ser referidos a centros con experiencia para el manejo de patología traqueal para el diagnóstico oportuno y el manejo multidisciplinario.

\section{REFERENCIAS}

1. Manninen MP, Antila PJ, Pukander JS, Karma PH. Occurrence of tracheal carcinoma in Finland. Acta Otolaryngol 1991;111(6):1162-1169.

2. Licht PB, Friis S, Pettersson G. Tracheal cancer in Denmark: a nationwide study. Eur J Cardiothorac Surg 2001;19(3):339-345.

3. Honings J, van Dijck JA, Verhagen AF, van der Heijden HF, Marres HA. Incidence and treatment of tracheal cancer: 
a nationwide study in the Netherlands. Ann Surg Oncol 2007;14(2):968-976.

4. Urdaneta Al, Yu JB, Wilson LD. Population based cancer registry analysis of primary tracheal carcinoma. Am J Clin Oncol 2011;34(1):32-37.

5. Perelman MJ, Koroleva N. Surgery of the trachea. World J Surg 1974;18:16-25.

6. Rivas de Andrés JJ, de la Torre Bravos MM, Blanco Gonzalez S, Córdoba Peláez M, Miguélez Vara C, Sobrido González F. Tumores traqueales poco frecuentes: carcinoma adenoideo-quístico y adenoma de glándulas mucosas. Cir Esp 2000;67(4):391-393.

7. Maziak DE, Todd TR, Keshavjee SH, Winton TL, Van Nostrand P, Pearson FG. Adenoid cystic carcinoma of the airway: thirty-two-year experience. J Thorac Cardiovasc Surg 1996;112(6):1522-1531.

8. Travis WD, Brambilla E, Muller-Hermelink HK, Harris CC. Of the lung, pleura, thymoma, and heart. In: World Health Organization Classification of Tumors: Pathology and Genetics, IARC Presee, Lyon, France 2004. Access date: 2013 November 11. Available from: http://www.iarc.fr/ en/publications/pdfs-online/pat-gen/bb10/BB10.pdf

9. Scherl S, Alon EE, Karle WE, Clain JB, Khorsandi A, Urken ML. Rare tracheal tumors and lesions initially diagnosed as isolated differentiated thyroid cancers. Thyroid 2013;23(1):79-83.

10. Bhattacharyya N. Contemporary staging and prognosis for primary tracheal malignancies: a population-based analysis. Otolaryngol Head Neck Surg 2004;131(5):639642.

11. Honings J, Gaissert HA, Verhagen AF, et al. Undertreatment of tracheal carcinoma: multidisciplinary audit of epidemiologic data. Ann Surg Oncol 2009;16(2):246-253.

12. Grillo HC, Mathisen DJ. Primary tracheal tumors: treatment and results. Ann Thorac Surg 1990;49(1):69-77.

13. Regnard JF, Fourquier P, Levasseur P. Result and prognostic factors in resections of primary tracheal tumors: a multicenter retrospective study. The French Society of Cardiovarcular Surgery. J Thorac Cardiovasc Surg 1996;111(4):808-813.
14. Gaissert HA, Grillo HC, Shadmehr MB, et al. Long-term survival after resection of primary adenoid cystic and squamous cell carcinoma of the trachea and carina. Ann Thorac Surg 2004;78(6):1889-1896.

15. Misiukiewicz KJ, Camille N, Tishler R, Haddad R, Limaye $\mathrm{S}$, Posner M. Organ preservation for adenoid cystic carcinoma of the larynx. Oncologist 2013;18(5):579-583.

16. Cavaliere S, Venuta F, Foccoli P, Toninelli C, La Face B. Endoscopic treatment of malignant airway obstructions in 2,008 patients. Chest 1996;110(6):1536-1542.

17. Colt HG, Harrell JH. Therapeutic rigid bronchoscopy allows level of care changes in patients with acute respiratory failure from central airway obstruction. Chest 1997;112(1):202-206.

18. Jeon K, Kim H, Yu CM, et al. Rigid bronchoscopic intervention in patients with respiratory failure caused by malignant central airway obstruction. J Thorac Oncol 2006;1(4):319-323.

19. Webb BD, Walsh GL, Roberts DB, Sturgis EM. Primary tracheal malignant neoplasms: the University of Texas MD Anderson Cancer Center experience. J Am Coll Surg 2006;202(2):237-246.

20. Suzuki T. What is the best management strategy for adenoid cystic carcinoma of the trachea? Ann Thorac Cardiovasc Surg 2011;17(6):535-538.

21. Pearson FG, Tood TR, Cooper JD. Experience with primary neoplasms of the trachea and carina. J Thorac Cardiovasc Surg 1984;88(4):511-518.

$\triangle$ Correspondencia:

Dr. Marco Antonio Iñiguez García, Jefe de la Clínica para la Atención de la Patología de la Tráquea y Bronquios Principales. Instituto Nacional de Enfermedades Respiratorias Ismael Cosío Villegas. Calzada de Tlalpan 4502, Colonia Sección XVI, México, D.F. 14080.

Teléfono: 015556668110

Correo electrónico: markcardio@hotmail.com

Los autores declaran no tener conflicto de intereses. 